\title{
DIAGNOSE FOLIAR DA GRAVIOLEIRA (Annona muricata L.): EFEITO DA POSIÇÃO DE RAMOS E FOLHAS
}

\author{
Part of plant to sample leaves for nutritional status evaluation in soursop (Annona muricata L.)
}

\author{
Rosiane de Lourdes Silva de Lima ${ }^{1}$, Gilvan Barbosa Ferreira ${ }^{2}$, Olmar Baller Weber ${ }^{3}$, Jairo Osvaldo Cazetta ${ }^{4}$
}

\begin{abstract}
RESUMO
A carência de informações na literatura sobre a amostragem de folhas da gravioleira, particularmente, a definição da posição da folha na copa das árvores e das folhas nos ramos ainda é um fator limitante para a diagnose foliar dessa cultura. Objetivando determinar a parte da planta indicada para a amostragem de folhas e a avaliação do estado nutricional da gravioleira conduziu-se um experimento no Campo Experimental da Embrapa Agroindústria Tropical, em Pacajus (CE), no período de março a abril de 2003. Colheram-se folhas de três posições da copa (terço superior, terço médio e terço inferior) e três posições no ramo (folhas da parte apical, mediana e basal) e determinaram-se os teores de $\mathrm{P}, \mathrm{K}, \mathrm{Ca}, \mathrm{Mg}, \mathrm{S}, \mathrm{Fe}, \mathrm{Cu}, \mathrm{Zn}$ e $\mathrm{Mn}$. Os resultados permitem sugerir que os teores de minerais das folhas da parte mediana da copa das árvores e na posição mediana do ramo refletem melhor o estado nutricional da frutífera.
\end{abstract}

Termos para indexação: Annona muricata, nutrição mineral, altura da copa.

\begin{abstract}
The lack of information in literature about leaf sampling soursop plant in particular, the definition of the leaf position at the tree top and leaves on the branches is still a limiting factor for the diagnosis of this cultivation. Aiming to determine which is the suitable part of the plant for leaf sampling and evaluate the nutritional status of the soursop plants, an experiment was developed on the experimental farm Embrapa - CNPAT, at Pacajus-CE, in the period from March to April, 2003. One collected leaves from three positions at the top (upper third, medium third and lower third) and three positions on the branches (apical part leaves, basal and medium) and one determined the tenor of $\mathrm{P}, \mathrm{K}, \mathrm{Ca}, \mathrm{Mg}, \mathrm{S}, \mathrm{Fe}, \mathrm{Cu}, \mathrm{Zn}$ and $\mathrm{Mn}$. The results allow to suggest that the mineral levels of the medium part of leaves at the top of the trees on the medium position of the branch, reflect best nutritional status of the plant.
\end{abstract}

Index terms: Annona muricata, mineral nutrition, position in the top of trees.

(Recebido em 12 de dezembro de 2006 e aprovado em 2 de abril de 2007)

\section{INTRODUÇÃO}

A análise foliar tem sido amplamente usada na diagnose do estado nutricional das plantas e baseia-se no fato de existir uma correlação direta entre a taxa de crescimento ou de produção e o teor de nutrientes nos seus tecidos (JONES et al., 1990). Dessa correlação são estabelecidas valores para os teores de nutrientes que correspondem às mudanças em termos de produção. Esses pontos representam níveis críticos e delimitam faixas de concentrações relacionadas às deficiências nutricionais, aos níveis adequados ou a toxidez de minerais (MARINHO et al., 2002).

A aplicação de métodos de interpretação para a diagnose do estado nutricional da gravioleira depara-se com problemas relacionados à ausência de consenso sobre qual parte da planta deve ser considerado na análise foliar. Existem vários fatores que podem determinar as variações nas concentrações de nutrientes em folhas, como por exemplo, a idade da folha e sua posição no ramo, desempenho da frutificação, cultivar, genótipo, tipo de solo e as práticas culturais. Assim, para avaliação do estado nutricional de plantas, torna-se relevante observar a posição da folha no dossel. Pesquisadores (AMARAL, 1998; KOO \& YOUNG, 1977) diagnosticaram o estado nutricional do abacateiro (Persea americana L.) utilizando folhas situadas na posição mediana do ramo. Em lichieira (Litchi chinensis Sonn.), Kotur \& Spingh (1993) recomendaram o segundo par de folhas a partir do ápice do ramo, em todas as direções e a meia altura da copa das árvores. Em aceroleira, Amaral (1998) utilizou a porção superior do dossel e os terços medianos e basais dos ramos. Para o mamoeiro, Marinho et al. (2002) constataram que o limbo foliar é mais sensível, quando comparado ao pecíolo, para avaliar o estado nutricional dessa cultura.

Informações básicas sobre a nutrição mineral da gravioleira são escassas, principalmente no que se refere à determinação da parte da planta indicada para avaliar o 
estado nutricional. Nesse sentido, objetivou-se neste trabalho avaliar a variação das concentrações de nutrientes em diferentes partes da copa e dos ramos da gravioleira, visando amostragem para diagnóstico do estado nutricional da planta.

\section{MATERIAL E MÉTODOS}

O estudo foi realizado num pomar de gravioleiras (Annona muricata L.) com 2,5 anos de idade, instalado numa área de Neossolo Quartzarênico Distrófico, no Campo Experimental da Embrapa Agroindústria Tropical, em Pacajus (CE), no período de março a abril de 2003. O solo possuía baixa fertilidade, apresentando acidez leve $(\mathrm{pH}$ $5,6)$ e presença de alumínio trocável em baixo teor $(1,2 \mathrm{mmol}$ $\left.\mathrm{dm}^{-3}\right)$; a saturação de bases apresentava-se relativamente baixa (57\%), baixo teor de $\mathrm{P}\left(215 \mathrm{mg} \mathrm{dm}^{-3}\right)$ e baixo teor de matéria orgânica $\left(11,48 \mathrm{~g} \mathrm{~kg}^{-1}\right)$.

As amostras de folhas da gravioleira foram coletadas em três posições da copa (terço superior, terço médio e terço inferior) e três posições no ramo (folhas da parte apical, mediana e basal). O delineamento foi o de blocos casualisados, com três repetições. Cada unidade experimental foi representada por 10 gravioleiras, que se encontravam em fase vegetativa, com 3 anos de idade, sendo obtidas por enxertia e cultivadas em condições de sequeiro.

As folhas após a coleta foram lavadas com água desionizada e postas para secar em estufa, sob ventilação forçada, a $65^{\circ} \mathrm{C}$, até atingir peso constante, e em seguida, foram moídas em moinho tipo Wiley, passadas em peneira de 20 mesh e homogeneizadas.

Posteriormente, essas amostras foram submetidas à digestão nítrico-perclórica para determinação dos teores de P, K, Ca, Mg, S, Fe, Cu, Zn e Mn. O P foi determinado pelo método da redução do fosfomolibdato pela vitamina C, modificado por Braga \& Defelipo (1974); o K, por fotometria de chama; o $\mathrm{Ca}, \mathrm{Mg}, \mathrm{Fe}, \mathrm{Cu}, \mathrm{Zn}$ e $\mathrm{Mn}$, por espectrofotometria de absorção atômica; e o S, por turbidimetria do sulfato (BLANCHAR et al., 1963).

Os dados foram analisados no delineamento de blocos casualisados, em esquema de parcelas subdivididas, sendo as posições da copa consideradas parcelas e as posições do ramo, as subparcleas. As médias dos tratamentos foram comparadas, aplicando-se o teste de Tukey a $1 \%$ e $5 \%$ de probabilidade, segundo Santos \& Ghey (2003).

\section{RESULTADOS E DISCUSSÃO}

Os teores de $\mathrm{P}, \mathrm{K}, \mathrm{Ca}, \mathrm{Mg}, \mathrm{S}$, Mn e $\mathrm{Zn}$ variaram dentre as posições da copa e do ramo da gravioleira (Tabela 1), o que já era esperado. Porém não houve interação dos fatores para teores de $\mathrm{Cu}$ e $\mathrm{Fe}$ nas folhas.

Os teores de $\mathrm{P}$ (Tabela 2) presentes nos tecidos foliares das gravioleiras encontram-se abaixo das faixas de 1,6 a 2,0 $\mathrm{g}$ $\mathrm{kg}^{-1}$, de 1,4 a 1,5 $\mathrm{g} \mathrm{kg}^{-1}$ e de 1,4 a 9,0 $\mathrm{g} \mathrm{kg}^{-1}$, consideradas adequadas por Andrade (2004), Oliveira (2004) e Robinson (1988), respectivamente, para plantas adultas de Annona spp. Destaque-se o teor de $\mathrm{P}$ no solo $\left(215 \mathrm{mg} \mathrm{dm}^{-3}\right.$ ), sendo considerado adequado, o que indica a necessidade de se estabelecer novos padrões para a cultura, na região.

Para diagnosticar o estado nutricional de fruteiras tais como gravioleira, Oliveira (2004) recomenda coletar 100 folhas completamente maduras (de 25 plantas por área homogênea), colhidas em ramos com flores, na altura média da copa das árvores. Já, Robinson (1988) recomenda que sejam coletadas 40 folhas recém-amadurecidas (a quarta ou quinta folha a partir do ápice do ramo), sem especificar a altura da copa, porém colhida nos meses em que as plantas estão em pleno florescimento. Os dados do presente trabalho confirmam a indicação da coleta na parte mediana da copa das árvores e nas folhas da posição mediana do ramo por apresentarem menor variação em plantas com 3 anos de idade e em estado vegetativo.

TABELA 1 - Análise de variância para teores de P, K, Ca, Mg, S, Cu, Fe, Mn e Zn nas folhas de gravioleira em função da posição no ramo e da parte da copa das árvores. Pacajus, CE, 2003.

\begin{tabular}{|c|c|c|c|c|c|c|c|c|c|c|}
\hline F.V & GL & $\mathbf{P}$ & $\mathbf{K}$ & $\mathrm{Ca}$ & Mg & $\mathbf{S}$ & $\mathrm{Cu}$ & $\mathbf{F e}$ & Mn & Zn \\
\hline Blocos & 2 & $0,51 \mathrm{~ns}$ & $0,19 \mathrm{~ns}$ & $0,6 \mathrm{~ns}$ & $0,18 \mathrm{~ns}$ & $0,02 \mathrm{~ns}$ & $3,71 \mathrm{~ns}$ & $8,68 \mathrm{~ns}$ & $490,26 \mathrm{~ns}$ & $24,90 \mathrm{~ns}$ \\
\hline Alt. da copa & 2 & $1,4 * *$ & $17,32 * *$ & $127 * *$ & $4,66 * *$ & $2,41 * *$ & $57,58 * *$ & $7,72 \mathrm{~ns}$ & $79306,30 * *$ & $444,30 * *$ \\
\hline Pos. no ramo & 2 & $0,6 \mathrm{~ns}$ & $1,28 *$ & $8,5 \mathrm{~ns}$ & $0,37 \mathrm{~ns}$ & $0,04 \mathrm{~ns}$ & $17,65 \mathrm{~ns}$ & $14,34 \mathrm{~ns}$ & $13177,40 * *$ & $21,30 \mathrm{~ns}$ \\
\hline Alt x Posição & 4 & $1,1 * *$ & $5,67 * *$ & $20 * *$ & $0,96^{*}$ & $0,18^{*}$ & $14,72 \mathrm{~ns}$ & $5,04 \mathrm{~ns}$ & $24282,52 * *$ & $293,50 * *$ \\
\hline Resíduo & 16 & 0,21 & 0,26 & 2,8 & 0,23 & 0,02 & 7,17 & 6,34 & 1930,15 & 10,61 \\
\hline
\end{tabular}

* significância e o nível de $5 \%$ e ** $1 \%$ no teste F. 
TABELA 2-Teores de P, K, Ca, Mg e S em folhas de diferentes posições do ramo e da copa da gravioleira. Pacajus, CE, 2003.

\begin{tabular}{|c|c|c|c|c|}
\hline \multirow{2}{*}{ Parte na copa } & \multicolumn{3}{|c|}{ Posição da folha no ramo } & \multirow{2}{*}{ Média } \\
\hline & Apical & Mediana & Basal & \\
\hline \multicolumn{5}{|c|}{ Teor de $\mathbf{P}\left(\mathrm{g} \mathrm{kg}^{-1}\right)$} \\
\hline Terço superior & $1,30 \mathrm{aA}$ & $0,99 \mathrm{aB}$ & $0,98 \mathrm{bB}$ & 1,09 \\
\hline Terço médio & $1,09 \mathrm{aA}$ & $1,14 \mathrm{aA}$ & $1,26 \mathrm{bA}$ & 1,13 \\
\hline Terço inferior & $1,24 \mathrm{aB}$ & $1,20 \mathrm{aA}$ & $1,59 \mathrm{aA}$ & 1,73 \\
\hline $\mathrm{CV} \%$ & 12,11 & & & \\
\hline \multicolumn{5}{|c|}{ Teor de K $\left(\mathrm{g} \mathrm{kg}^{-1}\right)$} \\
\hline Terço superior & $10,00 \mathrm{bB}$ & $10,57 \mathrm{aA}$ & $10,64 \mathrm{aA}$ & 10,4 \\
\hline Terço médio & $10,71 \mathrm{aA}$ & $10,41 \mathrm{aA}$ & $9,76 \mathrm{bB}$ & 10,3 \\
\hline Terço inferior & $9,23 \mathrm{cB}$ & $9,77 \mathrm{bA}$ & $9,72 \mathrm{bA}$ & 9,5 \\
\hline $\mathrm{CV} \%$ & 1,61 & & & \\
\hline \multicolumn{5}{|c|}{ Teor de $\mathrm{Ca}\left(\mathrm{g} \mathrm{kg}^{-1}\right)$} \\
\hline Terço superior & $7,57 \mathrm{bA}$ & $6,92 \mathrm{bA}$ & $6,92 \mathrm{bA}$ & 7,1 \\
\hline Terço médio & $7,44 \mathrm{bA}$ & $7,02 \mathrm{bA}$ & 7,68abA & 7,3 \\
\hline Terço inferior & $9,21 \mathrm{aB}$ & $10,55 \mathrm{aA}$ & $8,17 \mathrm{aB}$ & 9,3 \\
\hline $\mathrm{CV} \%$ & 6,65 & & & \\
\hline \multicolumn{5}{|c|}{ Teor de $\mathrm{Mg}\left(\mathrm{g} \mathrm{kg}^{-1}\right)$} \\
\hline Terço superior & $2,80 \mathrm{aA}$ & $2,77 \mathrm{aA}$ & $2,56 \mathrm{bA}$ & 2,7 \\
\hline Terço médio & $2,27 \mathrm{baB}$ & $2,25 \mathrm{bA}$ & $2,57 \mathrm{bA}$ & 2,4 \\
\hline Terço inferior & $2,62 \mathrm{aB}$ & $2,81 \mathrm{aAB}$ & $2,94 \mathrm{aA}$ & 2,8 \\
\hline $\mathrm{CV} \%$ & 5,75 & & & \\
\hline \multicolumn{5}{|c|}{ Teor de $S\left(\mathrm{~g} \mathrm{~kg}^{-1}\right)$} \\
\hline Terço superior & $1,22 \mathrm{aA}$ & $1,26 \mathrm{aA}$ & $1,06 \mathrm{aB}$ & 1,1 \\
\hline Terço médio & $0,93 \mathrm{bA}$ & $0,92 \mathrm{bA}$ & $0,98 \mathrm{abA}$ & 0,9 \\
\hline Terço inferior & $0,87 \mathrm{bA}$ & $0,83 \mathrm{bA}$ & $0,88 \mathrm{bA}$ & 0,8 \\
\hline $\mathrm{CV} \%$ & 5,00 & & & \\
\hline
\end{tabular}

Médias seguidas de uma mesma letra minúscula nas colunas e maiúsculas nas linhas não diferem entre si pelo teste de tukey a $5 \%$ de probabilidade.

Os teores foliares de $\mathrm{K}$ variaram entre as posições da copa e do ramo (Tabela 2). O maior acúmulo de $\mathrm{K}$ foi verificado nas folhas colhidas da posição mediana dos ramos e localizado no terço médio da copa. Quanto ao efeito da posição da folha no ramo, observaram-se variações significativas com teor máximo de $10,7 \mathrm{~g} \mathrm{~kg}^{-1}$, no terço médio da copa, e mínimo de $9,2 \mathrm{~g} \mathrm{~kg}^{-1}$ na porção mediana da copa.

$\mathrm{O} \mathrm{K}$ tem alta mobilidade no floema das plantas (MARSCHNER, 1995) e em situações de deficiência do elemento no solo podem-se ter sintomas típicos nas folhas mais velhas. Tal fato provavelmente não ocorreu neste trabalho, pois as concentrações de K, no terço inferior da copa das árvores variaram de $9,7 \mathrm{~g} \mathrm{~kg}^{-1}$, nas folhas colhidas das posições basais e medianas do ramo, a 9,2 $\mathrm{g} \mathrm{kg}^{-1}$ nas folhas mais jovens. Esses teores encontram-se na faixa de insuficiência proposta por Robinson (1988) (10,0 e 15,0 g $\mathrm{kg}^{-1}$ ) e inferiores aos diagnosticados por Oliveira (2004) $\left(26 \mathrm{~g} \mathrm{~kg}^{-1}\right)$. Andrade (2004) considera como padrões para folhas maduras e completamente desenvolvidas, localizadas na altura média da copa das plantas em ramos com flores, teores variando entre 18 a $26 \mathrm{~g} \mathrm{~kg}^{-1} \mathrm{de} \mathrm{K}$.

Os teores de $\mathrm{Ca}$ variaram amplamente dentro das posições da copa das árvores estudadas e entre as posições da folha no ramo (Tabela 2). As folhas localizadas na parte apical dos ramos e no terço mediano da copa das 
árvores tinham menores teores do elemento $\left(7,44 \mathrm{~g} \mathrm{~kg}^{-1}\right)$, quando comparados aos valores diagnosticados nos terços superiores $\left(7,57 \mathrm{~g} \mathrm{~kg}^{-1}\right)$ e inferiores $\left(9,21 \mathrm{~g} \mathrm{~kg}^{-1}\right)$ da copa das plantas. Por outro lado, os teores de $\mathrm{Ca}$ obtidos nas folhas coletadas das posições medianas e basais do ramo e situadas nos terços superior da copa das árvores foram os mais baixos.

Ao se comparar o efeito das posições das folhas no ramo dentro de cada terço da copa das árvores, verificaramse diferenças significativas apenas entre o terço inferior e o terço mediano da copa das árvores, constatando-se maior teor de $\mathrm{Ca}$ em folhas colhidas da posição mediana do ramo, localizado no terço inferior da copa $\left(10,55 \mathrm{~g} \mathrm{~kg}^{-1}\right)$. Para o Ca o local de preferência quanto ao acúmulo foi o terço inferior da copa das plantas, resultado contrário aos observados para o K, confirmando as diferenças existentes na mobilidade dos nutrientes na planta.

Os teores de $\mathrm{Ca}$, nos tecidos foliares da planta, estiveram na faixa indicada como adequada por Robinson (1988) situada entre 6 e $10 \mathrm{~g} \mathrm{~kg}^{-1}$, para plantas adultas de Annona spp e inferiores aos considerados padrões por Andrade (2004) e Oliveira (2004), que são de 8 a $17 \mathrm{~g} \mathrm{~kg}^{-1} \mathrm{e}$ de 12 a $18 \mathrm{~g} \mathrm{~kg}^{-1}$, respectivamente.

Maiores teores foliares de $\mathrm{Mg}$ (Tabela 2) ocorreram em folhas obtidas da posição basal do ramo, localizado no terço inferior da copa da planta $\left(2,9 \mathrm{~g} \mathrm{~kg}^{-1}\right)$, valores mínimos de $(2,2 \mathrm{~g}$ $\mathrm{kg}^{-1}$ ) ocorreram no tecido foliar obtido da posição apical do ramo, localizado no terço mediano da copa das árvores.

Para o $\mathrm{Mg}$, a posição da folha no ramo foi menos importante do que a localização na copa das árvores. Em duas posições do ramo (mediana e basal), a concentração do elemento aumentou do terço médio para cima ou para baixo na copa das plantas. Assim, a amostragem feita nos terços médios e superiores da copa das árvores sejam a mais adequada, pois se evitaria a medição dos teores acumulados no terço inferior e que não se redistribui para outros órgãos das plantas (MARSCHNER, 1995).

Os teores de magnésio apresentaram-se abaixo da faixa de 3,5 e 5,0 $\mathrm{g} \mathrm{kg}^{-1}$, considerada adequada por Robinson (1988); ou do valor 4,0 $\mathrm{g} \mathrm{kg}^{-1}$ mencionado por Oliveira (2004), ou ainda da faixa de 3,0 a 4,0 $\mathrm{g} \mathrm{kg}^{-1}$ citada por Andrade (2004). As variações dos teores de $\mathrm{Mg}$, nas folhas colhidas em diferentes posições no ramo, diferiram significativamente.

Para o S não ocorreram diferenças significativas nas folhas de distintas posições do ramo e da copa das plantas (Tabela 1). De forma geral, os teores desse nutriente variaram de 0,8 a $1,2 \mathrm{~g} \mathrm{~kg}^{-1}$, (Tabela 2), encontrando-se abaixo da faixa de 1,5 a $1,7 \mathrm{~g} \mathrm{~kg}^{-1}$ e de 1,6 a $2,0 \mathrm{~g} \mathrm{~kg}^{-1}$ considerada por Andrade (2004) e Oliveira (2004), respectivamente. Uma vez que os teores foliares de $S$ foram similares, em todas as partes da planta, esse elemento não seria o limitante no que se refere à eleição de uma parte da planta a ser amostrada.

Para o $\mathrm{Cu}$, (Tabela 3) as diferenças ocorreram apenas na posição do ramo, obtendo-se valores máximos de $15 \mathrm{mg}$ $\mathrm{kg}^{-1} \mathrm{em}$ tecidos foliares colhidos da posição apical do ramo e mínimo de $11 \mathrm{mg} \mathrm{kg}^{-1}$, observados na parte inferior da copa das árvores. Verificou-se similaridade entre os teores desse nutriente nas diversas partes da copa das árvores. Entretanto, baseado na excessiva sensibilidade dos teores de $\mathrm{Cu}$ na região meristemática da folha, que pode provocar teores baixos ou altos relacionados com restrições periódicas no solo à umidade, e na baixa translocação desse nutriente no floema (MARSCHNER, 1995), as folhas da posição mediana e do terço médio da copa das árvores são mais indicadas para fins de diagnosticar o estado nutricional das plantas.

Os teores de $\mathrm{Cu}$ observados encontram-se acima dos teores citados como suficientes por Andrade (2004), que menciona teores médios de $10 \mathrm{mg} \mathrm{kg}^{-1}$ para folhas maduras e completamente desenvolvidas na altura média da copa das árvores, em ramos com flores.

Quanto ao ferro não houve diferença significativa para os fatores estudados (Tabela 1). Os teores de Fe apresentaram evidências de se distribuir homogeneamente em todas as regiões analisadas. Isso dá indicação de que as folhas, de qualquer parte da planta, poderiam ser usadas para avaliar-se o teor de Fe, na cultura. Mas, os valores médios observados para o elemento (Tabela 3 ) encontram-se abaixo dos teores citados como suficientes por Andrade (2004), que menciona teores de $100 \mathrm{mg} \mathrm{kg-}^{1}$, para plantas de Annona spp.

Em relação ao Mn das folhas houve diferenças para as posições no ramo e para a parte na copa das árvores (Tabela 3). No terço superior da copa ocorreu maior concentração desse nutriente em tecidos foliares obtidos da posição basal do ramo $\left(626 \mathrm{mg} \mathrm{kg}^{-1}\right)$. Contudo, para a parte mediana e inferior da copa das árvores, os maiores teores do elemento ocorreram na posição apical dos ramos. Esse comportamento não era esperado, uma vez que o nutriente se acumula no tecido de ramos mais velhos (JONES JUNIOR et al., 1991).

Para o Zn, observou-se efeito significativo da interação entre a posição da folha no ramo e parte da copa das árvores (Tabela 1). Os teores máximos desse nutriente foram observados em tecidos foliares obtidos da posição apical do ramo da parte superior da copa (Tabela 3). Teores ligeiramente superiores foram detectados ainda na posição apical do ramo e na parte superior da copa das árvores. Ao contrário do observado para os macronutrientes, a posição das folhas no ramo é importante para diagnosticar o $\mathrm{Zn}$. 
LIMA, R. de L. S. de et al.

TABELA 3 - Teores de $\mathrm{Cu}, \mathrm{Fe}, \mathrm{Mn}$ e Zn em folhas de diferentes posições do ramo e da copa da gravioleira.

\begin{tabular}{|c|c|c|c|c|}
\hline \multirow{2}{*}{ Parte na copa } & \multicolumn{3}{|c|}{ Posição da folha no ramo } & \multirow{2}{*}{ Média } \\
\hline & Apical & Mediana & Basal & \\
\hline \multicolumn{5}{|c|}{ Teor de $\mathrm{Cu}\left(\mathrm{mg} \mathrm{kg}^{-1}\right)$} \\
\hline Terço superior & $16,49 \mathrm{aA}$ & $12,28 \mathrm{aA}$ & $11,68 \mathrm{aA}$ & 13,48 \\
\hline Terço médio & $17,49 \mathrm{aA}$ & $17,70 \mathrm{aA}$ & $12,60 \mathrm{aA}$ & 15,93 \\
\hline Terço inferior & $10,39 \mathrm{aA}$ & $10,53 \mathrm{aA}$ & $11,69 \mathrm{aA}$ & 10,87 \\
\hline CV\% & 19,93 & & & \\
\hline \multicolumn{5}{|c|}{ Teor de Fe $\left(\mathrm{mg} \mathrm{kg}^{-1}\right)$} \\
\hline Terço superior & $73,47 \mathrm{aA}$ & $75,52 \mathrm{Aa}$ & $72,36 \mathrm{aA}$ & 73,781 \\
\hline Terço médio & $72,85 \mathrm{aA}$ & $73,42 \mathrm{aA}$ & $72,33 \mathrm{aA}$ & 72,86 \\
\hline Terço inferior & $72,22 \mathrm{aA}$ & $76,73 \mathrm{aA}$ & $75,21 \mathrm{aA}$ & 74,72 \\
\hline $\mathrm{CV} \%$ & 3,41 & & & \\
\hline \multicolumn{5}{|c|}{ Teor de $\mathrm{Mn}\left(\mathrm{mg} \mathrm{kg}^{-1}\right)$} \\
\hline Terço superior & $447,85 \mathrm{bB}$ & $449,47 \mathrm{bB}$ & $625,98 \mathrm{aA}$ & 508 \\
\hline Terço médio & $744,35 \mathrm{aA}$ & $671,08 \mathrm{aAB}$ & $590,85 \mathrm{aB}$ & 669 \\
\hline Terço inferior & $529,87 \mathrm{bA}$ & $430,94 \mathrm{bB}$ & $553,04 \mathrm{aA}$ & 518 \\
\hline CV\% & 7,83 & & & \\
\hline \multicolumn{5}{|c|}{ Teor de $\mathrm{Zn}\left(\mathrm{mg} \mathrm{kg}^{-1}\right)$} \\
\hline Terço superior & $84,22 \mathrm{aA}$ & $74,09 \mathrm{aB}$ & $59,67 \mathrm{bC}$ & 73 \\
\hline Terço médio & $56,16 \mathrm{bB}$ & $59,36 \mathrm{bB}$ & $66,60 \mathrm{aA}$ & 61 \\
\hline Terço inferior & $58,51 \mathrm{bB}$ & $56,73 \mathrm{bB}$ & $65,60 \mathrm{abA}$ & 61 \\
\hline CV\% & 5,04 & & & \\
\hline
\end{tabular}

Médias seguidas de uma mesma letra minúscula nas colunas e maiúsculas nas linhas não diferem entre si pelo teste de tukey a $5 \%$ de probabilidade.

Contrastando-se os teores foliares de $\mathrm{Zn}$ obtidos na posição apical, mediana e basal dos ramos verificou-se menor concentração de Zinco, em valores médios, naqueles tecidos procedentes da região do terço médio da copa das árvores. A maior variação média foi de 56 a $84 \mathrm{mg} \mathrm{kg}^{-1}$ nas folhas localizadas na posição apical do ramo, e as menores diferenças foram entre as folhas do ramo da posição basal (60 a $66 \mathrm{mg} \mathrm{kg}^{-1}$ ).

Por um lado, a alta sensibilidade de variação nos teores de $\mathrm{Zn}$, nas folhas apicais, tende a confundir a interpretação do estado nutricional, pois as condições de campo são naturalmente variáveis e a baixa sensibilidade das folhas basais pode dificultar um diagnóstico de deficiência em condições limitantes de suprimento pelo solo, pois se sabe que o Zn é imóvel no floema (MARSCHNER, 1995). Assim, as folhas na posição mediana daqueles ramos, localizados no terço médio da copa, são as que devem ser indicadas para amostragem.
Os teores de Zn observados, encontram-se acima dos teores citados na literatura como suficientes por Robinson (1988), que os situa entre 15 e $30 \mathrm{mg} \mathrm{kg}^{-1}$, e por Andrade (2004) que menciona como padrão teores médios de $16 \mathrm{mg} \mathrm{kg}^{-1}$, para plantas adultas de annona spp.

\section{CONCLUSÕES}

As folhas da parte mediana da copa das árvores, na posição mediana do ramo, refletem adequadamente os teores dos nutrientes na gravioleira, sendo elas as mais indicadas para amostragem e diagnóstico do estado nutricional da planta.

\section{REFERÊNCIAS BIBLIOGRÁFICAS}

AMARAL, J. F. T. do. Parte da planta e época para diagnose do estado nutricional e crescimento de ramos em aceroleira (Malpighia emarginata D.C.). 1998. Dissertação (Mestrado em Fitotecnia) - Universidade Federal de Viçosa, Viçosa, 1998. 
ANDRADE, L. R. M. Corretivos e fertilizantes para culturas perenes e semiperenes. In: SOUSA, D. M. G.; LOBATO, R. (Eds.). Cerrado: correção do solo e adubação. 2. ed. Brasília, DF: Embrapa Informação Tecnológica, 2004. p. 317-366.

BLANCHAR, R. W.; REHM, G.; CALDWELL, A. C. Sulphur in plant materials by digestion whit nitric perchloric acid. Soil Science Society American Proceedings, Madison, v. 29, p. 71-72, 1963.

BRAGA, J. M.; DEFELIPO, B. V. Determinação espectrofotométrica de $\mathrm{P}$ em extratos de solo e material vegetal. Revista Ceres, Viçosa, v. 21, n. 113, p. 73-85, 1974.

JONES, J. B.; ECK, H. V.; VOSS, R. Plant analysis as an aid in fertilizing corn and grain sorghum. In: WESTERMAN, R. L. (Ed.). Soil testing and plant analysis. Madison: SSSA, 1990. p. 521-549.

JONES JUNIOR, J. B.; WOLF, B.; MILL, H. A. Plant analysis handbook, a pratical samplig, preparation, analysis, and interpretation guide. Anthens: Micro-Macro, 1991. p. 144.

KOO, R. C. J.; YOUNG, T. W. Effects of age, position, and fruiting status on mineral composition of 'Tonnage' avocado leaves. Journal American Society for
Horticultural Science, Alexandria, v. 102, n. 3, p. 311-313, 1977.

KOTUR, S. C.; SPINGH, H. P. Leaf-sampling technique in litchi (Litchi chinensis). Indian Journal of Agricultural Sciences, New Delhi, v. 63, n. 10, p. 632-638, 1993.

MARINHO, C. S.; MONNERAT, P. H.; CARVALHO, A. J. R. C.; MARTINS, S. L. D.; VIEIRA, A. Análise química do pecíolo e limbo foliar como indicadora do estado nutricional dos mamoeiros 'solo' e 'formosa'. Scientia Agrícola, Piracicaba, v. 59, n. 2, p. 373-381, 2002.

MARSCHNER, H. Mineral nutrition of higher plants. London: Academic, 1995. 888 p.

OLIVEIRA, S. A. Análise foliar. In: SOUSA, D. M. G.; LOBATO, R. (Eds.). Cerrado: correção do solo e adubação. 2. ed. Brasília, DF: Embrapa Informação Tecnológica, 2004. p. 245-366.

ROBINSON, J. B. Fruits, vines and nuts. In: REUTER, D. J.; ROBINSON, J. R. (Eds.). Plant analysis: an interpretation manual. Melbourne: Inkata, 1988. p. 120-147.

SANTOS, J. W.; GHEY, H. R. (Eds.). Estatística experimental aplicada. Campina Grande: Marcone, 2003. 213 p. 